\title{
Factors Governing The Behavior of Multiple Cooperating Swarms
}

\author{
Mohammed El-Abd \\ Electrical and Computer Engineering Dept. \\ University of Waterloo \\ 200 University Ave. W. \\ Waterloo, Ontario, Canada \\ mhelabd@pami.uwaterloo.ca
}

\author{
Mohamed Kamel \\ Electrical and Computer Engineering Dept. \\ University of Waterloo \\ 200 University Ave. W. \\ Waterloo, Ontario, Canada \\ mkame@pami.uwaterloo.ca
}

\begin{abstract}
This paper investigates the idea of having multiple swarms working separately and cooperating with each other to solve an optimization problem. Many factors that influence the behavior of this approach haven't been properly studied. This paper investigates two factors that affect this approach behavior. These factors are: (i) the communication strategy adopted if the number of swarms is raised above two, and (ii) the number of cooperating swarms. Experiments run on different benchmark optimization functions show that adopting a circular communication strategy gives better results than just sharing the global best of all the swarms. Increasing the number of cooperating swarms provides better results provided that the appropriate synchronization period is selected.
\end{abstract}

\section{Categories and Subject Descriptors}

I.2 [Computing Methodologies]: Artificial Intelligence; G.1.6 [Numerical Analysis]: Optimization

\section{General Terms}

Algorithms, Performance

\section{Keywords}

Particle swarm optimization, Cooperative search, Non-linear function optimization

\section{INTRODUCTION}

The area of cooperative search algorithms has been widely investigated in the past decade. There are many definitions in the literature for cooperative search. One definition is given in [1] "Cooperative search consists of a search performed by agents that exchange information about states, models, entire sub-problems, solutions, or other search space

Copyright is held by the author/owner.

GECCO'05, June 25-29, 2005, Washington, DC, USA.

ACM 1-59593-010-8/05/0006. characteristics". Furthermore, the authors also indicate that cooperative algorithms could be efficient even if they are sequentially implemented. Cooperative search has been proposed using Tabu Search [2], Genetic Algorithms [3], and Ant Colony [4].

Particle Swarm Optimization (PSO) [5] is a stochastic optimization technique that was originally developed to simulate the behavior of a flock of birds or a school of fish looking for food.

\section{PARTICLE SWARM OPTIMIZATION}

The PSO method is regarded as a population-based method, where the population is referred to as a swarm. The swarm consists of a number of individuals called particles. The first model used is the gbest, in which each particle moves toward its own achieved personal best and the best position achieved by the whole swarm.

\section{COOPERATION IN PSO}

A cooperative PSO approach that relies on explicit space decomposition was introduced in [6]. The approach relies on splitting the space (solution vector) into sub-spaces (smaller vectors) where each sub-space is optimized using a separate swarm. The overall solution is the vector containing the best particle of each swarm.

A concurrent approach that relies on implicit space decomposition was recently introduced in [7]. This approach is based on having two swarms searching concurrently for a solution with frequent message passing of information. One swarm adopted the original PSO method, and the other used the Fitness-To-Distance Ratio PSO FDRPSO [8]. This approach improved the performance over both methods as well as minimizing the time requirement of the FDRPSO alone. Other cooperative approaches were also proposed in $[9,10]$.

In [11], the affect of changing the synchronization period was studied. Results showed that this approach improved the solution quality over the one obtained by a single swarm in multimodal functions only. Experiments also showed that the implementation technique adopted, sequential or parallel, does not change this model behavior.

\section{EXPERIMENTAL RESULTS}

The experiments were run using two multimodal benchmark test functions, namely the rastrigin, and the ackley functions. 
In these experiments, the cooperating swarms have 10 particles each, and adopt the gbest model. The space dimensionality is 10. 100000 function evaluations are carried out in every run. The results reported are the averages taken over 50 different runs.

\subsection{Communication Strategy}

Two different communication strategies is being tested in this work: (i) sharing the global minimum among all swarms, (ii) circular communication of the global minimum. Experiments are run using three swarms. Figure 1 shows the results.

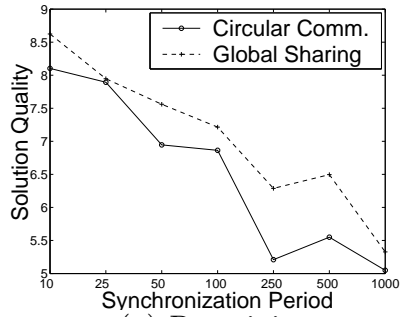

(a) Rastrigin

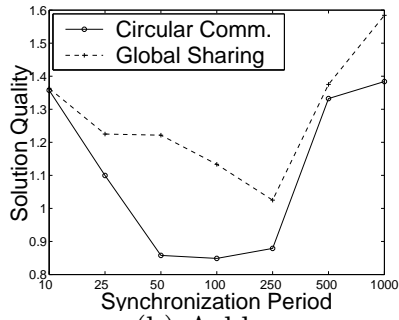

(b) Ackley
Figure 1: Results of applying different communication strategies

The results show that the circular communication approach gives systematically better results than the global sharing approach. This is due to the diversity maintained using this approach.

\subsection{Number of Swarms}

When comparing the performance of the cooperative PSO model with different number of swarms, the number of particles will be kept fixed. This means that increasing the number of cooperating swarms will lead to having less number of particles per swarm. The experiments are run using two, three, four then five cooperating swarms adopting circular communication. The number of particles is always kept fixed at 30. Results shown in figure 2 .

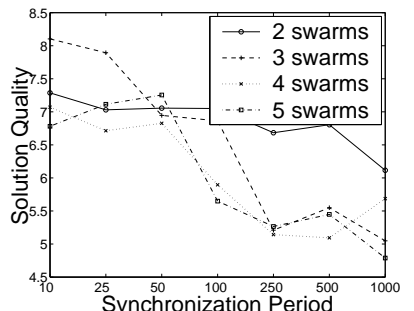

(a) Rastrigin

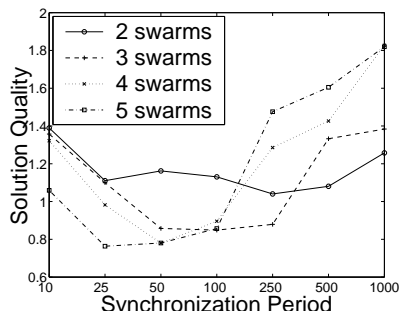

(b) Ackley
Figure 2: Results of increasing the number of swarms

In both functions, increasing the number of swarms led to improve the solution quality provided that the suitable synchronization period is selected.

\section{CONCLUSIONS}

Several experiments are run to test the affect of the communication strategy and the number of cooperating swarms on the performance of a multiple cooperating swarms technique.

The results show that increasing the number of cooperating swarms above two is beneficial, provided that the suitable synchronization period is selected. The circular communication strategy is proven to give better results when having more than two cooperating swarms.

Another issue to be addressed in future work is communicating different type of information, namely the best particles in the swarms instead of just the global best.

\section{REFERENCES}

[1] M. Milano and A. Roli, "Magma: A multiagent architecture for metaheuristics," IEEE Transactions on Systems, Man, and Cybernetics, Part B: Cybernetics, vol. 33, no. 2, 2004.

[2] T. G. Crainic and M. Gendreau, "Cooperative parallel tabu search for capacitated network design," Journal of Heuristics, vol. 8, pp. 601-627, 2000.

[3] M. Nowostawski and R. Poli, "Parallel genetic algorithm taxonomy," in 3rd International Conference on Knowledge-Based Intelligent Information Engineering Systems, 1999, pp. 88-92.

[4] M. Middendorf, F. Reischle, and H. Schmeck, "Information exchange in multi colony ant algorithms," in Proceedings of 15 IPDPS 2000 Workshops, 3rd Workshop on Biologically Inspired Solutions to Parallel Processing Problems (BioSP3), 2000, pp. 645-652.

[5] J. Kennedy and R. C. Eberhart, "Particle swarm optimization," in Proceeding of the IEEE

International Conference on Neural Networks, vol. 4, 1995, pp. 1942-1948.

[6] F. van der Bergh and A. P. Engelbrech, "A cooperative approach to particle swarm optimization," IEEE Transactions on Evolutionary Computation, vol. 8, no. 3, pp. 225-239, 2004.

[7] S. Baskar and P. N. Suganthan, "A novel concurrent particle swarm optimization," in Proceedings of the 2004 Congress on Evolutionary Computation, vol. 1, June 2004, pp. 792-796.

[8] T. Peram, K. Veeramachaneni, and C. K. Mohan, "Distance-fitness-ratio particle swarm optimization," in Proceeding of the IEEE 2003 Swarm Intelligence Symposium, 2003, pp. 174-181.

[9] T. Blackwell and J. Branke, "Multi-swarm optimization in dynamic environments," in Applications of Evolutionary Computing, vol. 3005 LNCS Springer, pp. 488-599, 2004.

[10] C. A. C. Coello, "Using clustering techniques to improve the performance of a multi-objective particle swarm optimizer," in Lecture Notes in Computer Science, vol. 3102 Springer-Verlag, pp. 225-237, 2004.

[11] M. El-Abd and M. Kamel, "Mutiple cooperating swarms for non-linear function optimization," in Accepted in 2nd Intenartional Workshop for Swarm Intelligence and Patterns, 4th Inetrnational IEEE Workshop in Soft Computing as Transdisciplinary Science and Technology, 2005. 